\title{
A Comparative Study of the Two Translation Versions of Qiu Chuang Feng Yu Xi from the Perspective of Skopos Theory
}

\author{
GAO Yufeng \\ Wuhan University of Technology, Wuhan, 430070 China
}

\begin{abstract}
Hong Lou Meng is one of the most important works in Chinese classical novels, and the author, Cao Xueqin, gave his own understanding of the feudal society and his exclamation of the fickleness of the world in the novel. Up to now, the novel and the poems in the novel are still of high artistic value. Qiu Chuang Feng Yu Xi, as one of the most artistic and representative poems in the novel, has been translated into English by many translators. Among the translations, Yang Xianyi’s translation and David Hawkes’ translation have the highest artistic achievements. Yang Xianyi is a famous Chinese translator and David Hawkes is a world-renowned sinologist in Britain. Because of their distinct identities, their translation purposes and translation styles are totally different. Guided by German translator Hans Vermeer's Skopos Theory, this paper investigates the prologues of the translations of Hong Lou Meng and the translators' biographies. The findings indicate the respective translation purposes of Yang Xianyi and David Hawkes and their trade-off choices of translation methods. By means of objectively evaluation of the merits and demerits of the two translation versions of Qiu Chuang Feng Yu Xi, the rationality of existence of the translations in a specific cultural background is explained. It is proved that Skopos Theory is one of the general methods for the English translation of poems in Chinese classical novels.
\end{abstract}

Keywords: skopos theory, Qiu Chuang Feng Yu Xi, translation purpose, translation method

\section{Introduction}

\subsection{The Author and His Poetry}

Cao Xueqin, a great novelist of Qing Dynasty, was born in Jiangning, Jiangsu Province (now Nanjing, Jiangsu Province), and his ancestral home is Liaoyang, Shengjing Area (now Liaoyang, Liaoning Province). Cao Xueqin experienced a rich and romantic life in Jiangning in his early years. In the 6th year of the reign of Emperor Yongzheng (1728), Cao Xueqin moved to Beijing with his family after his family was confiscated for its crimes. During this period, Cao Xueqin completed the world-famous Chinese classical novel "Hong Lou Meng”. Cao Xueqin died in Beijing in the 28th year of the reign of Emperor Qianlong (1763). Cao Xueqin's master work, Hong Lou Meng, is widely recognized as the peak of Chinese classical novels because of its large scale, rigorous structure, complicated plot, and vivid description.

The poetry “Dai Bie Li, Qiu Chuang Feng Yu Xi” (hereinafter referred to as Qiu Chuang Feng Yu Xi) was written by Cao Xueqin, in the 45th chapter of the Chinese classical novel "Hong Lou Meng”. In the novel, the

GAO Yufeng, Undergraduate Student, School of Foreign Languages, Wuhan University of Technology, China. 
poetry was written by Lin Daiyu in the style of Chun Jiang Hua Yue Ye, the poetry of Zhang Ruoxu. Surrounded by the word "qiu (autumn)", the whole poetry shows the melancholy and sorrow of the helpless girl, Lin Daiyu. This poetry, linking scenery with emotion, is highly praised by many literary researchers. Zhou Ruchang, a famous scholar of Hong Lou Meng, believed that Qiu Chuang Feng Yu Xi had made the highest artistic achievements in three poems of Lin Dai Yu: Zang Hua Yin, Qiu Chuang Feng Yu Xi, and Tao Hua Xing. Among them, the last two poems were romantic and unrestrained, beautiful and sad, and far above the first one (Zhou, 2004).

\subsection{The Translators: Yang Xianyi and David Hawkes}

Yang Xianyi, a famous Chinese translator, foreign literature researcher and poet, was born in Tianjin in 1915. In the early 1960s, Yang Xianyi and his wife Gladys Yang (a British scholar of Chinese culture), began to translate Hong Lou Meng into English, which was completed in 1974. Their translation, called A Dream of Red Mansions, had a huge impact. On November 23, 2009, Yang Xianyi died in Beijing at the age of 95. As a translation expert of Beijing Foreign Languages Press, Yang Xianyi actively responded to the Chinese government's plan of “translating the classics into English”. Yang Xianyi once criticized Arthur Waley's translation of The Book of Songs for portraying the peasants of China's Zhou Dynasty as the medieval peasants of Europe, so that the translation reads more like an English medieval folk song than a poem about China (Ren, 1993, p. 34). Yang Yi, Yang Xianyi’s sister, once pointed out that Yang Xianyi and Gladys Yang had translated many classics, not because of their interests, but because they thought it was necessary to introduce China's cultural heritage to the outside world, and they thought foreigners should know how rich China's cultural heritage is (Yang, 1986, p. 41). Therefore, it can be found that, in the process of translating Hong Lou Meng into English, Yang Xianyi always aimed at exporting national cultural characteristics and establishing national image.

David Hawkes, a world-renowned sinologist, was born in east London in 1923. In 1970, David Hawkes resigned his job at University of Oxford and devoted himself to the English translation of Hong Lou Meng. David Hawkes spent 10 years translating the first 80 chapters of Hong Lou Meng, while his son-in-law, John Minford, a sinologist, translated the last 40 chapters. The translation, called The Story of the Stone, had a huge impact. David Hawkes died on July 31, 2009, aged 86. According to David Hawkes, the translator is responsible for the author, the text and the reader, and the three are often hard to reconcile (Hawkes, 1977, p. 20). David Hawkes once sighed, "If I could give the reader one percent of the pleasure I had in reading this Chinese novel, it would be worth living.” (Hawkes, 1973, p. 46 ). Obviously, to make the English translation of Hong Lou Meng acceptable better to the recipient in the target language and cultural background, has become the translation purpose of David Hawkes.

\section{Overview of Skopos Theory}

Skopos Theory was proposed by German translation theorist Hans Vermeer in the 1970s and was introduced to China by domestic scholars in the 1980s. The word "skopos” is Greek, meaning "purpose”. Hans Vermeer believes that translation is an action with a certain purpose. The purpose is determined by the initiator of the translation action, while the way to achieve the purpose is determined by the translator, so the translator in the translation process is crucial (Vermeer, 2000, p. 221). After Hans Vermeer, Christiane Nord 
systematically summarized the viewpoints of Functional Translation Theory and proposed "function and loyalty” as a supplement to Skopos Theory (Nord, 2001).

The use of Skopos Theory should conform to three rules: Skopos Rule, Coherence Rule, and Fidelity Rule. The translator's translation strategy must be determined by the purpose or the function of the translation, which is called "Skopos Rule". While paying attention to the function of the translation, Skopos Theory also emphasizes the readability of the translation in the target language environment, namely "Coherence Rule", and the inter-textual coherence between the translation and the original, namely "Fidelity Rule".

\section{Comparative Analysis of the Two Translation Versions Based on Skopos Theory}

\subsection{In Wording}

According to different translation purposes, Yang Xianyi and David Hawkes adopted different wording methods in the English translations of Qiu Chuang Feng Yu Xi. In the translations of the following four lines and the title of the poetry, Yang Xianyi and David Hawkes obviously adopted different wording methods. This part will take the following two examples as the analysis objects:

Example (1)

Source Text: 泪烛摇摇蓺短檠, 诨愁照恨动离情。

Yang Xianyi’s Version: Guttering on its stick, the candle sheds tears of wax, evoking the grief of separation, its pain.

David Hawkes’ Version: The candle weeps down to its socket low, and my heart weeps and desolation feels.

“泪烛” is a classic image in Chinese classical poetry, and is often used to create lonely and sad scenes. In the translation of these two lines, in order to better introduce Chinese traditional culture to the west, Yang Xianyi chose the method of literal translation, using "guttering” and “tears” to depict the scene of “泪烛摇摇”, which is faithful to the original. Obviously, Yang Xianyi's translation successfully exports national cultural characteristics to the west. By contrast, David Hawkes chose the method of free translation, using the word "weeps", to depict the scene of a candle melting into tears and a woman weeping over the candle, which is more acceptable to the audience in the target language and cultural background, and more creative than Yang Xianyi's translation.

Furthermore, Yang Xianyi translated these two lines in the third person, whereas David Hawkes translated these two lines in the first person. David Hawkes used the word "my" with great empathy. Obviously, in the translations of these two lines, both Yang Xianyi and David Hawkes chose the appropriate wording methods based on their respective translation purposes under the guidance of Skopos Rule.

Example (2)

Source Text: 罗袰不奈秋风力, 残漏声催秋雨急。

Yang Xianyi’s Version: The autumn wind, through silken quilts strikes chill, her water-clock the autumn rain spurs on.

David Hawkes' Version: The wind's chill strikes through quilt and counterpane, the rain drums like a mad clock in my ears. 
Obviously, both Yang Xianyi's translation and David Hawkes' translation convey the literal meaning of this sentence, but David Hawkes ignored the translation of “罗袰”. “罗袰” refers to a quilt made of silk. In ancient China, only the wealthy could afford it. Yang Xianyi chose "silken” to reflect the material of making “罗袰”, which more accurately conveyed the meaning of the original.

Besides, in order to make western readers understand his translation better, David Hawkes used "drums" and "mad clock" to describe the noise of rain like a mad clock, which made people crazy. Obviously, David Hawkes mistranslated the original. By contrast, the word "water-clock" in Yang Xianyi's translation better conveys the artistic conception of the original. It is apparent that compared with David Hawkes' version, Yang Xianyi followed Fidelity Rule in his translation by paying much attention to inter-textual coherence between the translation and the original.

To sum up, in terms of wording, the translation methods chosen by Yang Xianyi and David Hawkes are obviously different. Yang Xianyi focused on literal translation based on his translation purpose, while David Hawkes focused on free translation based on his translation purpose. Yang Xianyi's translation is faithful to the original and can better convey Chinese culture to the western world, but its wording is not as creative as David Hawkes' translation. In contrast to Yang Xianyi's version, the wording of David Hawkes' translation is more flexible and can be better accepted by western readers, but it abandons the beautiful artistic conception of the original to some extent.

\subsection{In Sentence Pattern}

According to different translation purposes, Yang Xianyi and David Hawkes adopted different sentence pattern methods in the English translations of Qiu Chuang Feng Yu Xi. In the translations of the following four lines of the poetry, Yang Xianyi and David Hawkes obviously adopted different sentence pattern methods. This part will take the following two examples as the analysis objects:

Example (3)

Source Text: 秋花惨淡秋草黄, 耿耿秋灯秋夜长。

Yang Xianyi’s Version: Sad the autumn flowers, sear the autumn grass, autumn lamps thicker through the long autumn night.

David Hawkes' Version: The autumn flowers are dead; the leaves are sere; lamp-light comes soon; the nights grow long again.

In these two lines of the original, the author used the sentence pattern of "noun + adjective" to describe autumn flower, autumn grass, and autumn night, so as to highlight the lonesome season, autumn. According to his translation purpose, Yang Xianyi used the sentence pattern of “adjective + noun" to depict autumn flower, autumn grass, and autumn night, which is faithful to the original to the greatest extent. By contrast, David Hawkes changed the sentence pattern and used four short sentences of "subject + verb + predicative" to depict autumn flower, autumn leaf, autumn lamp-light and autumn night. It can be seen that David Hawkes' translation is concise and powerful, which flexibly handled the sentence pattern of the original. Obviously, in the translations of these two lines, both Yang Xianyi and David Hawkes chose the appropriate sentence pattern methods to better convey the spirit of the original to their recipients, which conforms to their perspective translation purposes.

Example (4) 
Source Text: 助秋风雨来何速？惊破秋窗秋梦绿。

Yang Xianyi’s Version: The wind and rain speed autumn on its way, by the window shattering her autumn dream.

David Hawkes' Version: The rustling rain came in such swift downpour, it startled me from autumn-dream-filled sleep.

In the original, the author lamented how quickly the autumn wind and autumn rain came with the sentence pattern of interrogative sentence. In translations, although Yang Xianyi and David Hawkes both translated the literal meaning of these two lines from the sentence pattern of declarative sentence, neither adopted the sentence pattern of interrogative sentence like the original nor the sentence pattern of exclamatory sentence guided by "how". It can be seen that their translations detract from the beauty of the original to a certain extent, resulting in the failure of both to achieve their respective translation purposes. Obviously, both Yang Xianyi and David Hawkes violate the inter-textual coherence between the translation and the original.

It can be found that in terms of sentence pattern, Yang Xianyi was more faithful to the original sentence pattern based on his translation purpose of exporting national cultural characteristics and establishing national image. By contrast, David Hawkes made some flexible treatment of the original sentence pattern based on his translation purpose of making the English translation of Hong Lou Meng acceptable better to the recipient in the target language and cultural background. Yang Xianyi's translation has a high degree of fidelity, while David Hawkes' translation has a high degree of flexibility. However, both Yang Xianyi and David Hawkes have some demerits in the translation of rhetorical question, which detract from the aesthetic conception of the original to some extent and obviously violate Fidelity Rule of Skopos Theory.

\subsection{In Prosody}

According to different translation purposes, Yang Xianyi and David Hawkes adopted different prosody methods in the translations of Qiu Chuang Feng Yu Xi.

In terms of rhyme, Qiu Chuang Feng Yu Xi, as a typical Chinese classical poetry, its rhyme is end rhyme. There are five stanzas in Qiu Chuang Feng Yu Xi, each having four lines. The rhyme of the original is "aaba” for the first four stanzas, and "abcb" for the last stanza. As for David Hawkes' translation, in all five stanzas of the poetry, rhyme is "abab". However, in Yang Xianyi's translation, there is no rhyme in the first and second stanzas, the rhyme of the third and fourth stanzas is "abcb", and the rhyme of the fifth stanza is "abab".

Seen from David Hawkes' translation, in terms of rhyme, it conforms to iambic pentameter, which is common in English poetry. In other words, David Hawkes successfully handled his translation with British characteristics based on his translation purpose. By contrast, the rhyme of Yang Xianyi's translation does not reproduce the style of the original. It can be seen that, the first and second stanzas of Yang Xianyi's translation have no rhyme, which is extremely inconsistent with the style of Chinese classical poetry. So Yang Xianyi's translation does not achieve his translation purpose. Therefore, in terms of rhyme, the achievement of David Hawkes' translation is higher than Yang Xianyi’s translation.

Regarding the repetition of single word, “秋” is reused 15 times in the original. The repeated use of the word “秋” renders the context of autumn wind and autumn rain. In the translations, Yang Xianyi used “autumn” 14 times to translate “秋”, whereas David Hawkes only used “autumn” 3 times to translate “秋”. “秋” is pronounced 
"qiu” in Chinese, and has fewer syllables than "autumn”. Needless to say, by comparison, David Hawkes' translation is more concise than Yang Xianyi's translation, while Yang Xianyi's translation is more faithful than David Hawkes' translation.

It can be found that in terms of the repetition of single word, under the guidance of Skopos Theory, Yang Xianyi's translation is faithful to the original, while David Hawkes' translation deviates from the original. Yang Xianyi's translation is not concise enough, which violates intra-textual coherence to some extent. David Hawkes' translation is not faithful enough, which violates inter-textual coherence to some extent.

\section{Findings and Suggestions Proposed}

According to the above comparative research results, the following findings and suggestions based on Skopos Theory applicable to the translation of Chinese classical poetry can be proposed.

First of all, the purpose of translation should be made clear before translating. Secondly, in translation process, specific translation methods should be selected according to specific translation purpose and various contextual factors.

In terms of wording, if the purpose of translation is centered on the original, the wording should be given priority to literal translation, and the cultural elements associated with the original should be preserved in the translation. If the purpose of translation focuses on the recipient, the wording should be given priority to free translation, and the acceptability of the wording in the target language environment should be paid attention to. Whatever the purpose of translation is, the translator should pay attention to the veracity of wording, avoid errors and omissions in translation, and make the translation conform to Coherence Rule and Fidelity Rule to the greatest extent.

In terms of sentence pattern, if the purpose of translation is given high priority to the original, the sentence pattern of translation should be as faithful as possible to the original. If the purpose of translation is centered on the recipient, the sentence patterns of the translation can be flexibly to a certain extent according to the language habits of the recipient. Whatever the purpose of translation is, the translator should try his best to be faithful to the original when dealing with the sentence pattern of question, the sentence pattern of rhetorical question and other special sentence patterns.

In terms of prosody, the translator should select the appropriate prosody pattern according to his specific translation purpose. Furthermore, the translator should decide to reserve or reject the special prosody patterns of the original in the translation based on his specific translation purpose.

\section{Conclusion}

Skopos Theory, as an important part of Functional Translation Theory, is a powerful theoretical tool in translation studies. From the perspective of Skopos Theory, the purpose of the whole translation action is the most important factor in the translation process. In the two English translation versions of Qiu Chuang Feng Yu $X i$, Yang Xianyi took the original as the center, so he adopted the method of literal translation, whereas David Hawkes took the recipient as the center, so he adopted the method of free translation. Both translation versions have been praised and loved by different groups of readers. This demonstrates the scientific nature of Skopos Theory in guiding translation action. 
It can be concluded that the evaluation of the value of a translation cannot be separated from the translator's translation purpose and the translation's social background. The above comparative study unfolds that the translator should choose appropriate wording methods, sentence pattern methods, and prosody methods in the translation process according to his translation purpose. Poetry in Chinese classical novels is regarded as an excellent carrier of Chinese history and culture. Properly translating Chinese classical poetry will be conductive to the spread of Chinese culture and the cross-cultural communication between China and the world.

\section{References}

Hawkes, D. (1973). The story of the stone I. London: Penguin Books.

Hawkes, D. (1977). The story of the stone II. London: Penguin Books.

Nord, C. (2001). Translating as a purposeful activity-functionalist approaches. Shanghai: Shanghai Foreign Language Education Press.

Vermeer, H. (2000). Skopos and commission in translational action. In Lawrence, V (Ed.), The translation studies reader (pp. 221-232). London and New York: Routledge.

任生名. (1993). 杨宪益的文学翻译思想散记. 中国翻译, (4), 33-35.

周汝昌. (2004). 红楼夺目红. 北京: 作家出版社.

曹雪芹, 高鹗. (2004). 红楼梦. 长沙:岳麓书社.

杨茨. (1986). 杨宪益与翻译. 中国翻译, (5), 40-41. 